lungs, IL-13 suppressed Adam33 mRNA but no difference in $a$-smooth muscle actin (aSma) was evident. Immunoblotting for ADAMA33 in BALF demonstrateda $76 \mathrm{kDa}$ band, consistent with the ADAM33 ectodomain and processed forms at $38 / 44 \mathrm{kDa}$ in $\mathrm{dTg}$ animals. ADAM33 enzymatic activity was also significantly increased.

Conclusion The data suggest thatallergic inflammation induced by IL-13 suppresses Adam33 mRNA expression but induces the release of soluble forms ofADAM33, yielding enzymatically active forms. The release of soluble forms may play a role in airway remodelling, potentiallyleading to BHR. We next propose to test the effect of specific ADAM33inhibitors on airway remodelling in this allergic mouse model to assess theirpotential as novel treatments for asthma.

\section{S119 ROLES OF TLR3, TLR4- AND TLRS7-9 IN INTERFERON INDUCTION IN BRONCHIAL EPITHELIAL CELLS AND PERIPHERAL BLOOD MONONUCLEAR CELLS FROM ASTHMATIC AND NON-ASTHMATIC SUBJECTS}

doi:10.1136/thoraxjnl-2012-202678.124

${ }^{1} \mathrm{~A}$ Sykes, ${ }^{2} \mathrm{MR}$ Edwards, ${ }^{1} \mathrm{~J}$ Macintyre, ${ }^{1} \mathrm{~A}$ del Rosario, ${ }^{1} \mathrm{OM}$ Kon, ${ }^{1} \mathrm{SL}$ Johnston. ${ }^{1} / \mathrm{mperial}$ College London and Imperial Healthcare NHS Trust, London, UK; ${ }^{2}$ Imperial College London, London, UK

Introduction Defective rhinovirus (RV) induced interferon (IFN)- $\beta$ and IFN- $\lambda$ production has been reported in primary human bronchial epithelial cells (HBECs) and peripheral blood mononuclear cells (PBMCs) from asthmatics. The mechanisms of defective IFN induction in asthma are unknown. Virus infection can induce IFNs through Toll like Receptors (TLR)3, TLR4 and TLRs7-9 and TLR agonists have been identified as potential therapeutic options for asthma. The role of these TLRs in IFN induction in asthma is unclear. Objective To investigate IFN responses to TLR stimulation in HBECs and PBMCs from atopic asthmatic and non-asthmatic individuals.

Methods HBECs and PBMCs from atopic asthmatic and nonasthmatic subjects were stimulated with agonists to TLR3, TLR4 \& TLRs7-9 and type I and III IFN responses assessed by qPCR and ELISA.

Results TLR3 and TLR7, but not TLR4, 8 or 9, stimulation induced IFN protein and mRNA expression in HBECs and PBMCs. IFNs induced were IFN- $\beta$ and predominantly type III IFN- $\lambda$ in HBECs and type I ( $-\alpha$ and $-\beta)$ with no IFN- $\lambda$ in PBMCs. TLR function was not defective in asthmatic compared to non-asthmatic subjects.

Conclusions TLR3 \& TLR7 were the predominant TLRs involved in IFN induction in HBECs and PBMCs. Defective IFN induction to TLR agonists was not observed in these well controlled asthmatic subjects. TLR3/7 agonists could be effective in inducing IFNs in more severe/less well controlled asthmatics who may have deficient virus induced IFN production.

\section{Evaluation and treatment of Cystic Fibrosis}

\section{S120 UPDATE ON THE UK CF GENE THERAPY CONSORTIUM MULTIDOSE, NON-VIRAL, GENE THERAPY TRIAL}

doi:10.1136/thoraxjnl-2012-202678.125

'EWFW Alton, 'D Ashby, ${ }^{2} \mathrm{C}$ Boyd, ${ }^{3} \mathrm{~S}$ Cheng, ${ }^{4} \mathrm{~S}$ Cunningham, JC Davies', ${ }^{5} \mathrm{D}$ Gill, ${ }^{1} \mathrm{U}$ Griesenbach, ${ }^{1} \mathrm{~T}$ Higgins, ${ }^{5} \mathrm{~S}$ Hyde, ${ }^{6} \mathrm{JA}$ Innes, ${ }^{2} \mathrm{G}$ Murray, ${ }^{2} \mathrm{D}$ Porteous. ${ }^{1}$ Imperial College, London, UK; ${ }^{2}$ University of Edinburgh, UK; ${ }^{3}$ Genzyme Corp, Framingham, USA; ${ }^{4}$ Royal Hospital for Sick Children, Edinburgh, UK; ${ }^{5}$ University of Oxford, UK; ${ }^{6}$ Western General Hospital, Edinburgh, UK

The UK CF GTC has been working for several years to determine the clinical benefit of CFTR gene therapy. Our premise was that for such a therapy to achieve clinical benefit, repeated administration would be required, and that therefore a non-viral approach was needed. We demonstrated in laboratory and preclinical models that GL67A (Genzyme Corp) was the optimal gene transfer agent, and designed a plasmid, pGM169, completely depleted of proinflammatory $\mathrm{CpG}$ motifs and driven by the novel hCEFI promoter, designed for prolonged expression. In a longitudinal observational study (the Run-in) we measured the variability of multiple outcome measures, both conventional and novel. These data have allowed us to perform power calculations and a) choose our primary outcome $\left(\mathrm{FEV}_{1}\right)$, b) secondary efficacy outcomes (lung clearance index, various parameters on CT scan, Quality of life questionnaire [CFO-R], exercise capacity and activity, and selected sputum and serum inflammatory markers), and c) safety measures (clinical findings, exacerbation rate, gas transfer, sputum culture, serum inflammatory markers, renal and hepatic markers). We have recently completed a single-dose safety and dose ranging study.

In this trial, 130 patients, aged 12 years and above are being randomised in a 1:1 fashion to active treatment or placebo and will receive the nebulised agent at monthly intervals for 12 doses. The group size was determined on the basis of a $6 \%$ relative improvement in $\mathrm{FEV}_{1}$. An adaptive design will be used for additional safety; the first 20 patients will receive 3 doses ahead of the rest of the cohort. Patients will be invited to participate in either one or two substudies, being conducted to explore mechanisms; a) nasal administration followed by nasal potential difference (PD) and brushings for mRNA expression and b) pre and post-treatment bronchoscopies for lower airway $\mathrm{PD}$, gene expression and histology. The double-blinded nature of the trial means that final outcome data will only be available upon completion of the study. The trial was initiated in April 2012; here we will update on recruitment, projected time-lines and progress.

Funded by the MRC and NIHR through the EME programme.

\section{S121 LUNG CLEARANCE INDEX TO EVALUATE THE EFFECT OF IVACAFTOR ON LUNG FUNCTION IN SUBJECTS WITH CF WHO HAVE THE G551D-CFTR MUTATION AND MILD LUNG DISEASE}

doi:10.1136/thoraxjnl-2012-202678.126

${ }^{1} \mathrm{JCD}$ Davies, ${ }^{2} \mathrm{HS}$ Sheridan, ${ }^{3} \mathrm{PSL}$ Lee, ${ }^{3} \mathrm{TS}$ Song, ${ }^{3} \mathrm{AS}$ Stone, ${ }^{4} \mathrm{FR}$ Ratjen. ${ }^{1}$ Royal Brompton Hospital and Imperial College, London, UK; ${ }^{2}$ University of Edinburgh, Edinburgh, UK; ${ }^{3}$ Vertex Pharmaceuticals Incorporated, Cambridge, USA; ${ }^{4}$ The Hospital for Sick Children, Toronto, Canada

Background Ivacaftor has been shown to lead to significant improvement in lung function, exacerbation rate, weight gain and quality of life in adolescents and adults with CF and the G551DCFTR mutation.

Objectives Drugs targeting the basic defect of CF may hold potential for patients with early stage disease, but establishing benefit is more difficult. If $\mathrm{FEV}_{1}$ is normal, a more sensitive test may be useful. LCI has been shown to become abnormal at an earlier stage of disease than $\mathrm{FEV}_{1}$ and thus may be a more sensitive outcome measure in this group of patients.

Methods This Phase 2, randomised, double-blind, placebocontrolled, multicenter, crossover study evaluated the effect of ivacaftor on LCI derived from multibreath washout of $\mathrm{SF}_{6}$ using an Innocor device. Subjects were $\geq 6$ years with the G551D-CFTR mutation, $\mathrm{FEV}_{1}>90 \%$ predicted, and $\mathrm{LCI}>7.4$ (upper limit of nor$\mathrm{mal}$ ). Ivacaftor $150 \mathrm{mg}$ or placebo was administered twice daily for two 4-week periods with a 4-week washout in between.

Results Twenty-one subjects were randomised and 20 received a dose of ivacaftor. Seventeen subjects completed both periods. Mean (SD) age was 16.6 (10.9) years. Mean (SD) baseline LCI was 9.0 (1.5). The treatment effect of ivacaftor for adjusted mean change from baseline in LCI at Day 29 was $-2.1(P=0.0004)$. Mean 
(SD) baseline $\mathrm{FEV}_{1}$ was $97.2 \%$ predicted (10.6\%). Treatment difference for the mean change from baseline in $\mathrm{FEV}_{1}$ at Day 29 was 7.0 percentage points $(P=0.0117)$. Treatment difference for the mean change from baseline in sweat chloride at Day 29 was -45.9 $\mathrm{mmol} / \mathrm{L}(P<0.0001)$. Treatment effect for the change from baseline in pooled CFQ-R Respiratory Domain score was 4.1 points versus placebo $(P=0.4020)$. In the ivacaftor period, AEs and SAEs were reported in 13 and 2 subjects, respectively. In the placebo period, AEs and SAEs were reported in 15 and 1 subject, respectively.

Conclusions In CF patients with mild lung disease, ivacaftor improved ventilation inhomogeneity as measured by LCI and respiratory function as measured by percent predicted $\mathrm{FEV}_{1}$. LCI is more sensitive to change in this mildly affected group of subjects and may be a useful outcome measure for future interventional trials in such patients.

\section{S122 IS THERE A GENDER DIFFERENCE IN THE UK CF POPULATION?}

doi:10.1136/thoraxjnl-2012-202678.127

'N Baty, ${ }^{2} S J$ MacNeill, ${ }^{2 P}$ Cullinan, 'EWFW Alton, ${ }^{3} \mathrm{D}$ Bilton, ${ }^{1} \mathrm{U}$ Griesenbach. 'Department of Gene Therapy, Imperial College Londonthe UK CF Gene Therapy Consortium, London, United Kingdom; ' $D$ epartment of Occupational and Environmental Medicine, Imperial College London, London, United Kingdom; ${ }^{3}$ Adult CF Centre, Royal Brompton Hospital, London, United Kingdom

The literature is conflicting with regards to gender differences in the CF population with some studies reporting poorer prognosis in CF females. Variations in study design (cross-sectional $v$ longitudi$\mathrm{nal}$ and single $\mathrm{v}$ multi-centre), study populations (paediatric $\mathrm{V}$ adults) and small sample sizes may in part explain these differences. In an attempt to overcome some of these limitations we analysed the UK CF Trust Registry database, which collates data on the paediatric and adult UK CF population for evidence of gender differences.

Methods We used the 2010 annual review data and compared males and females in age-groups $0-5,6-12,13-15,16-19,20-23$, $24-29$ and $30+$ years for lung function ( $\mathrm{FEV}_{1}$ and $\mathrm{FVC}$ (\% predicted)), infection status, treatments (rhDNase, $\mathrm{O}_{2}$ therapy, Non-Invasive Ventilation (NIV), IV antibiotics, supplementary feeding and pancreatic supplements), body mass index and associated complications (CF related diabetes, osteoporosis, liver disease).

Results Of the 7937 patients attending annual review in 2010 47\% were female. The sex distribution differed by age whereby the proportion of females dropped from 16 years onwards (Figure 1). The mean FEV ${ }_{1}$ was significantly $(p<0.001)$ lower in female teens aged 16-19 years compared to males (females: 71.0 (confidence interval 68.8-73.2), males 76.7 (74.6-78.8), but not in any other age group. Similar results were observed for mean FVC for which females aged $20-23$ yrs also had significantly lower values than males. Teenage female salso had a significantly $(p<0.05)$ higher use of rhDNase, gastrostomies, $\mathrm{O}_{2}$, had more days/yr on IV antibiotics in hospital and had a significantly $(\mathrm{p} \leq 0.001)$ higher rate of CFRD. In addition, a significantly $(p<0.05)$ higher percentage of females older than 13 had more home IV antibiotics. We did not detect any gender differences with respect to chronic bacterial colonisation(PA, SA, Bcepacia and MRSA).

Conclusions There is evidence of a more severe lung function deficit in teenage years. The reduced proportion of females in the adult cohorts is consistent with a survival difference. Further longitudinal analyses will help characterise the identified gender gap and effects on disease progression.

We thank the UK CF Trust for the registry data.

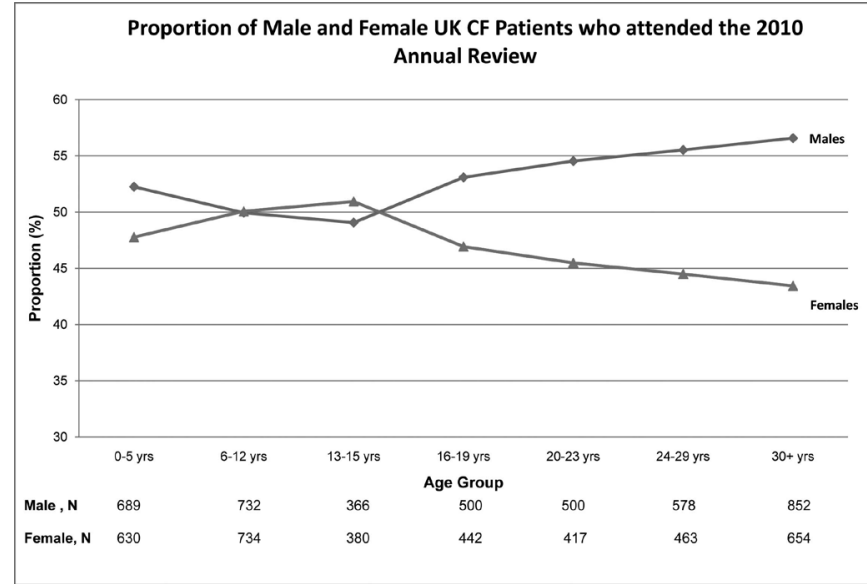

Abstract S122 Figure 1

\section{S123 THE ROLE OF NASAL POTENTIAL DIFFERENCE TESTING IN DIFFICULT CASES OF POSSIBLE CYSTIC FIBROSIS}

doi:10.1136/thoraxjnl-2012-202678.128

'N Simmonds, ${ }^{2} \mathrm{R}$ Pabary, ${ }^{1}$ Y Ya-Tung, 'D Bilton, ${ }^{2}$ EWFW Alton, ${ }^{2} \mathrm{JC}$ Davies. 'Royal Brompton and Harefield NHS Foundation Trust, London, UK; ${ }^{2}$ Imperial College, London, UK

The European CF Diagnostic Working Group guidelines include nasal potential difference (nPD) testing when conventional tests (sweat chloride/CFTR mutation analysis) are inconclusive. This specialist measure of epithelial ion transport is available in relatively few centres throughout the UK and Europe and therefore may not be readily considered as a diagnostic option. We have been offering a clinical service for several years, for both our own patients and those referred in. Here, we report the results of a retrospective audit over a 2.5 year period with an aim to increase awareness and understanding of the test.

Between March 2009 and Sept 2011, 66 patients (58\% female) underwent testing. Median (range) age was 18 (2-67) years. Children under the age of 8 were most commonly tested whilst under anaesthetic for a bronchoscopy. 59\% of patients were referred from other centres. The majority of sweat chloride results (52\%) were in the 30-60 mEq borderline range. 95\% had undergone first-line CFTR mutation analysis: $62 \%$ had none, $37 \%$ had one and 1 subject had 2 mutations, one of which was considered a 'sequence variant'.

One child was unable to tolerate the procedure; $6 \%$ of traces were uninterpretable due to presumed nasal inflammation. Overall, the $\mathrm{nPD}$ results in the context of other features led to a diagnosis of variant/atypical CF in 18\%; abnormality in chloride secretion was more sensitive than sodium absorption. We were able to rule out CF 'to the best of current diagnostic ability' in 60\% including 6 of 8 patients diagnosed with CF in early childhood, but now considered so unlikely to have the disease that re-investigation was warranted. In a further $13 \%$ of cases, nPD was of good quality but was difficult to categorise; in addition to the full CFTR gene sequencing performed routinely, we will investigate these patients for mutations in genes such as $\mathrm{ENaC}$.

Nasal PD was well tolerated and helpful in clarifying the diagnosis in the majority $(78 \%)$ of patients referred. It should be considered in patients for whom, despite careful work up, a diagnosis of $\mathrm{CF}$ is difficult to confirm or rule out.

\section{S124 THE ROLE OF PANCREATIC BETA-CELLS IN THE DIURNAL VARIATION OF GLUCOSE HANDLING IN CYSTIC FIBROSIS}

doi:10.1136/thoraxjnl-2012-202678.129

D Nazareth, K Mohan, M Walshaw. Liverpool Adult CF Unit, Liverpool, United Kingdom

Introduction An impaired glucose tolerance later in the day may be an early sign of diabetes ${ }^{1,2}$, suggesting that there is diurnal variation 